\title{
Civilisations
}

Revue internationale d'anthropologie et de sciences

humaines

$51 \mid 2004$

Religions transnationales

\section{Entrée en « modernité »}

A propos de : P.-J. LAURENT, Les pentecôtistes du Burkina Faso. Mariage, pouvoir et guérison, Paris, Karthala, 2003

\section{Frédéric Moens}

\section{(2) OpenEdition \\ Journals}

Édition électronique

URL : http://journals.openedition.org/civilisations/699

DOI : $10.4000 /$ civilisations. 699

ISSN : 2032-0442

\section{Éditeur}

Institut de sociologie de l'Université Libre de Bruxelles

\section{Édition imprimée}

Date de publication : 1 janvier 2004

Pagination : 193-200

ISSN : 0009-8140

Référence électronique

Frédéric Moens, "Entrée en « modernité » », Civilisations [En ligne], 51 | 2004, mis en ligne le 07 janvier 2009, consulté le 22 avril 2019. URL : http://journals.openedition.org/civilisations/699; DOI : 10.4000/ civilisations.699

Ce document a été généré automatiquement le 22 avril 2019

(C) Tous droits réservés 


\title{
Entrée en « modernité »
}

\author{
A propos de : P.-J. LAURENT, Les pentecôtistes du Burkina Faso. Mariage, \\ pouvoir et guérison, Paris, Karthala, 2003
}

\section{Frédéric Moens}

1 L'ouvrage de Pierre-Joseph Laurent recèle une profusion de données ethnographiques sur laquelle se bâtissent son exposé et son raisonnement. Cette multitude d'informations enrichit le lecteur d'une expérience qui, s'il l'acquiert par procuration, n'en est pas moins vivante; cette abondance constitue aussi pour lui une difficulté. En effet, le foisonnement de l'ouvrage en dilue, ou plus exactement en éclate, le propos : quel est le sujet de cette somme de plus de quatre cents pages? Certes, le titre parait explicite, il y est traité des pentecôtistes burkinabés. Cependant, cette évidence - par ailleurs, partiellement remise en cause par le sous-titre qui, parlant de "Mariage, pouvoir et guérison», ne souligne plus l'enracinement spatial - ne résiste pas à la lecture. De page en page, et ce dès l'introduction, le lecteur est entraîné dans des préoccupations bien plus génériques que l'implantation du pentecôtisme au Burkina Faso et, a fortiori, en Afrique de l'Ouest. D'aucuns diraient aussi que c'est bien moins que ce que le titre n'annonce puisque, en matière de pentecôtisme, seule une Eglise protestante est envisagée, les Assemblées de Dieu. Les justifications de ce choix, reposant avant tout sur la place que cette Eglise occupe au Burkina Faso tant en termes historiques qu'en termes numériques, sont rapidement posées. De qui est dressé le portrait? Est-ce celui de ce pays, à travers l'introduction au cours des quatre-vingts dernières années d'une nouvelle confession protestante? Est-ce celui de ce pentecôtisme, dans l'avatar que lui propose son incarnation dans un lieu particulier? Il me semble que, s'il faut choisir entre les deux branches de cette alternative, il est plus judicieux de s'en tenir à la première. Toute la chair de l'ouvrage est composée des transformations que ce pays sahélien a subies au cours des dernières années et la place, encore très marginale, qu'y occupe une Eglise protestante particulière, une place qui permet d'éclairer les stratégies individuelles dont elle est, elle-même, l'objet. Cette rencontre entre le bouleversement d'un pays et l'irruption d'une Eglise, qui met bien au centre le pays, est singulière car chacune des parties prenantes possédait des éléments la rendant apte à mieux s'adapter à l'autre. Je prendrai donc le pli dans cette brève note critique de lire cet ouvrage de la manière la 
plus large possible, démontrant qu'il s'agit, à travers l'exposé circonstancié et informé d'un cas monographique, de la volonté d'embrasser un problème central de l'anthropologie et de la sociologie contemporaines.

2 Cette question centrale n'est évidemment pas celle de l'histoire des Assemblées de Dieu ni même de l'histoire de presque un siècle de Haute Volta puis de Burkina Faso. Ce qui est très finement décrit, à travers le prisme d'un lieu et d'une stratégie religieuse, est la transformation de la société africaine en une nouvelle société, la disparition des références exclusivement paysannes ${ }^{1}$ et, en même temps, le fait que jamais les choses ne disparaissent totalement ou, au contraire, sans que les nouvelles choses, importées ou acceptées, ne soient elles-mêmes appropriées, accommodées. Comme tout espace social, l'Afrique, le Burkina Faso dans ce cas précis, demeure un lieu singulier (Bourdin, 2000) ; la modernité qui les frappe de front ne les détruit pas, elle les reconstruit. Rien ne s'y inscrit comme dans du sable, tout vient s'ajouter à une histoire, à un passé ; cette affirmation apparait, j'en conviens, d'une banalité consternante, et pourtant, tant dans nos collègues que dans les développeurs de tout ordre, la conviction est forte de pouvoir faire table rase du passé, de la culture ou, simplement, des us et coutumes. Ces préoccupations, parfois folkloriques, souvent essentielles, demeurent inévitablement en arrière-fond de toute transformation sociale. Débusquer non seulement ces éléments de pérennité mais aussi les stratégies individuelles et collectives mises en œuvre pour réaliser les accommodements nécessaires à l'accueil le moins destructeur de ces nouveautés exprime un projet d'ensemble de l'auteur (Laurent, 1998). A mon estime, si Les pentecôtistes au Burkina Faso renferme un concept central, pour l'auteur comme pour le lecteur, il s'agit de celui de « modernité insécurisée ». Plus loin dans cette note, je proposerai une critique de ce concept, il n'est à ce stade que question d'en proposer une première figure. A première vue, cette modernité insécurisée est une contradiction entre les aspirations des individus, les portant à une autonomie plus grande, à une émancipation des règles de la tradition, à une inscription dans les logiques anonymes de la modernité, et leurs moyens, toujours tellement précaires qu'ils ne peuvent leur permettre d'atteindre ces ambitions. Une telle définition, dont Laurent s'abstient bien intelligemment, nous rapproche aussi insensiblement que fortement de l'anomie durkheimienne dont l'origine est bien, souvenons-nous-en, dans cette disparité entre les espoirs et les possibilités des individus évoluant dans des sociétés "faibles ", des sociétés qui n'ont plus la puissance normative suffisante pour contraindre leurs ressortissants dans leurs ambitions (Durkheim, 1897 : 281). Ce qui structure donc le travail dont l'ouvrage nous fait le récit est la compréhension d'une stratégie emblématique de dépassement de cette anomie : faute de pouvoir y ajouter du sens, c'est de la cohérence qui y est adjointe.

3 Un paradoxe de l'exposé est de nous montrer la cohérence d'un monde naissant de façon parfois un peu incohérente, à tout le moins de manière déstructurée, à notre charge d'y retrouver, sans grand peine, l'ossature logique. La dizaine de chapitres ne parle jamais que de cette même chose : comment les hommes réintroduisent une cohérence dans leur action alors que leur monde se délite et que le monde nouveau qui leur est offert n'est pas encore réellement à leur taille. Pour nous faire intégrer cette réalité, il nous faut suivre à la fois l'arrivée de ce mouvement pentecôtiste dans ce pays et le contexte cultuel et culturel qu'il représente. Ces chapitres, qui remettent sur le métier les mêmes faits, parfois les mêmes personnages, font la richesse littéraire de l'ouvrage - rapidement nous faisons partie de la famille qui nous est décrite, comme l'auteur, nous les fréquentons longuement -, ils posent aussi quelques questions de style et de menus problèmes de 
répétition, la complexité de la structure narrative paraissant parfois avoir perdu son auteur qui nous redonne, de temps à autre, des passages quasi identiques dans la description d'un personnage, d'une situation, d'un fait social.

Avant d'aller plus loin, examinons rapidement dans son déploiement discursif l'argument qui nous est narré. Les soixante pages de la première partie, décrivent la rencontre particulière entre une culture et un contexte social, d'une part, et un type caractéristique de pentecôtisme, d'autre part. Moins que le succès et l'enracinement que les Assemblées de Dieu vont trouver en pays mossi pour essaimer dans la sous-région, Laurent nous montre l'enracinement rural d'origine, la vigueur que ces pasteurs-paysans donnent non seulement à leur foi mais aussi à sa mise en œuvre. Il démonte ainsi un premier syncrétisme: ce protestantisme s'ajoute, sans le modifier complètement, à une cosmogonie qui, pour les pasteurs et leurs fidèles, se renforce d'outils leur permettant d'agir dans cette histoire du $\mathrm{XX}^{\mathrm{e}}$ siècle qui laisse l'Afrique de l'Ouest face à de radicales transformations. Le chapitre consacré à la vocation de pasteur, à travers trois récits contemporains, raffermit la description historique du chapitre qui le précède; à une première vision macro-sociologique - les flux et les raisons de l'implantation de l'cEuvre ${ }^{2}$ - succède une seconde vision, qui la conforte, en montrant les logiques individuelles, micro-sociologique, de son succès. La deuxième partie, forte d'une centaine de pages, modifie insensiblement l'angle d'attaque : ce pentecôtisme offre à l'individu, cherchant à s'extraire des contraintes de la tradition, des stratégies d'action efficaces. La «famille en prière » ne se substitue pas à la famille sociale (et biologique) mais elle permet de s'en prémunir en y inscrivant différemment les mêmes mécanismes. Ainsi, cette «alliance protestante » intègre totalement la tradition en lui ajoutant une nouvelle strate ; au début des années 1990, un pasteur des Assemblées de Dieu m'expliquait à Loumbila que Jésus est une grosse pierre le protégeant, lui et les convertis de cœur, de tous les fétiches (Moens, 2002). La très fine et très longue description du mariage protestant montre certes l'attrait que le mouvement peut avoir pour les jeunes en rupture de ban traditionnel, mais elle dévoile aussi la force syncrétique à l'œuvre dans ce pentecôtisme. Composée elle aussi d'une grosse centaine de pages, la troisième partie dépeint avec le même souci du détail l'irruption des Assemblées de Dieu dans la sphère publique. Le premier chapitre de cette partie décrit de manière circonstanciée des séances d'évangélisation. Le prosélytisme pentecôtiste n'est pas une aporie: en effet, si ce protestant méprise autant qu'il craint l'espace public et la gestion du bien collectif - sa foi l'invite au contraire à s'occuper de son jardin, à fuir la politique qui n'est à ses yeux qu'une expression de la corruption du monde -, il lui est essentiel de prouver aux yeux de tous la force de ses convictions et, dans cet univers animiste, leur puissance : « [...] le prosélytisme des Assemblées de Dieu participe d'une pacification face à la violence engendrée par les multiples transformations de la société. [...] Je me convertis pour me protéger des 'autres', voire de moi-même d'abord, et je tente, ensuite, de convertir mon entourage, plutôt que de le tuer à la suite de la certitude que je me suis progressivement forgé qu'ils m'en veulent " (Laurent, $2003: 201$ ). Cette présence dans l'espace public n'est donc pas pour l'investir mais, au contraire, pour le prendre à témoin de son inutilité : le pasteur et ses fidèles s'exposent à tous pour leur montrer l'efficacité de leur conversion. Les scènes qui nous sont croquées décrivent les combats entre stratégies tentant d'accommoder les transformations de leur monde: le prosélytisme, par l'affirmation publique qu'il constitue, affermit autant les fidèles dans leur foi qu'il ne permet d'en convaincre de nouveaux. Le second chapitre de cette troisième partie expose la place que la politique tient aujourd'hui dans les Assemblées de Dieu. Longtemps volontairement séparés de la 
chose publique, les pasteurs ont été rattrapés par cette réalité. Soutiens implicites du pouvoir en place, qu'ils se refusent de juger mais pour lequel ils prient, leur succès et l'intégrité dont on les crédite ont contraint le mouvement à devenir un acteur politique. Incarnant la probité, fidèles et pasteurs se retrouvent à moraliser l'action publique par leurs témoignages. La suite de l'analyse de Laurent développe en quoi le pentecôtisme participe à la gestion des transformations des modes de régulation sociale de la société mossi. Cette régulation, qui nous est dite reposer sur une confiance familiale (kis-sida) et une entente villageoise (wuum taaba), se désagrège dans une modernité contemporaine où la volonté d'individuation et d'autonomie écarte toute possibilité d'une confiance familiale nécessairement contraignante et d'une entente villageoise obligatoirement équilibrée. Pour reprendre les termes de Laurent, alors que la "concorde coutumière " est en perdition, aucune "concorde civile» ne vient remettre la société à flot. Ce pentecôtisme réintroduit des cadres d'action permettant à la fois d'expliciter les déficiences des solidarités traditionnelles et d'en imposer, à leur échelle, de nouvelles. Partant de l'histoire du mouvement pour être de plus en plus plongée dans l'actualité des Assemblées de Dieu, la quatrième et dernière partie détaille leurs échecs et les solutions endogènes proposées. D'abord rurales, et telle était bien leur originalité, les Assemblées de Dieu burkinabées ont gagné les villes. Les difficultés personnelles comme les carences collectives de solidarité ne font ainsi que croître. Les mécanismes à l'œuvre dans le prosélytisme doivent être constamment réactivés, à travers diverses formes d'offre thérapeutique. Une fois encore, le travail de l'auteur est avant tout ethnographique : il rapporte avec talent et détails des séances de croyants-guérisseurs ou de thérapie collective. Ce qu'il montre ainsi sont des Assemblées de Dieu instituées qui, n'étant plus la radicale nouveauté, se trouvent confrontées à une contestation. Pour autant, les outils syncrétiques qu'elles ont développés - alliant les logiques de la tradition et celles supposées être de la modernité - demeurent et sont ceux qui lui sont opposés.

5 Accumulant les détails pour embrasser la logique d'une métamorphose sociale, la question posée tout au long de l'ouvrage est celle de la gestion sociale de la modernité. L'Afrique, le Burkina Faso vivent dans la même contemporanéité que le reste du monde ; les influences - économiques, sociales ou culturelles - qu'ils subissent sont isomorphes à celles qui frappent l'Europe, l'Asie ou les Amériques. Toutefois, si les formes sont comparables, l'incarnation qu'elles prennent et les conséquences qu'elles entraînent divergent de lieu en lieu, sous l'emprise des histoires locales. Les propos de l'introduction insistent sur ce point : l'objet du livre est la forme prise par la modernité dans une société africaine, nécessairement composition de son histoire, de ses influences et des contraintes contemporaines d'un monde globalisé : " Le temps de la 'modernité insécurisée' conjugue l'affaiblissement de la prise en charge coutumière de la vie commune et les difficultés de l'Etat d'organiser dans la sérénité la survie de pans entiers de sa population. Ainsi, [...] les dotations financières de l'Etat pour l'enseignement et la santé s'amenuisent et cèdent le pas aux services privés payants ${ }^{3}$ " (Laurent, $\left.2003: 21\right)$. La modernité actuelle, modernité avancée, n'est évidemment pas l'apanage d'un lieu ou d'un continent; elle pose à tous les individus et à tous les espaces sociaux des problèmes ${ }^{4}$. Laurent nous expose, comme centre de son observation, ces stratégies d'intégration de la nouveauté et d'hybridation des cultures.

6 La précarité de l'environnement fait des mécanismes de solidarité collective un élément vital de la survie individuelle et collective. Pour rendre compte de ces mécanismes, Laurent parle de concordes. La protection de l'individu, qu'elle soit matérielle ou 
symbolique, passe par les relations qu'il tisse et entretient avec ses proches, c'est-à-dire à travers des relations complexes de parenté. Cette protection a aussi un prix : l'individu ne peut s'extraire de la relation collective, il n'est en fait pas un sujet autonome mais la part d'un sujet collectif. S'affranchir de cette contrainte est une des ambitions les plus présentes, devenir un sujet individuel et autonome - sur le plan économique, sur celui des alliances que l'on lie ou, encore, de la vie que l'on mène - est le premier pas en «modernité ». Mais, sur un tel chemin, les embûches sont nombreuses: "On ne rit pas avec la parenté, elle aide. C'est une richesse, mais c'est aussi elle qui fait qu'on ne peut pas gérer de l'argent » (Laurent 2003 : 390). Cette citation de Zoundou Zoungrana, un des informateurs très présent dans le récit, modèle parfait de l'informateur dont Bourdieu fait l'éloge, à la fois dedans et dehors, résume pour partie le problème que pose Pierre Joseph Laurent : la famille protège mais enferme, elle est de façon coutumière un bouclier et de façon moderne un poids entravant tout développement (personnel, bien sûr, mais également ce développement social, polysémique et mythique ; $c f$. Rist, 1996 et Laurent, 1998).

7 Sans le porter à l'explicite, Laurent développe un chiasme intéressant : à la sécurité émasculante des principes coutumiers se substitue l'insécurité inhérente aux principes enivrants de la modernité. La modernité insécurisée, dont je persiste à croire que ce qu'il en dit sur l'Afrique peut s'appliquer aussi à d'autres lieux, n'est ainsi pas tellement éloignée de la concorde coutumière : l'accent est mis dans la première sur l'insécurité à laquelle la modernité se confronte alors que pour la seconde, ce qui est accentué est la résolution d'une insécurité endémique. Car, en effet, la concorde coutumière telle qu'elle nous est proposée repose non sur la loyauté et l'entente spontanée entre personnes mais sur un équilibre de la terreur, un équilibre où le respect d'autrui, l'accord entre chacun, la réciprocité de tous sont le résultat d'une contrainte sociale alliant un don agonistique à une méfiance parfaitement répandue de tous envers chacun. L'insécurité de la modernité réside dans la disparition de cette entraide lignagère et dans l'absence de l'émergence d'un substitut étatique : l'entourage coutumier n'est plus un support et il n'est plus une contrainte, mais l'individu qui s'est émancipé de ces chânes s'est aussi privé de ces ressources. La fuite en avant ou le saut dans le vide demande alors qu'une autre entente puisse s'établir. Une stratégie pour transcender ce dilemme réside dans le choix religieux. Par le choix d'un travail sur soi, d'une conversion à une croyance tout à la fois intégrante et structurante, la liberté personnelle acquise se trouve renforcée et l'incertitude ambiante partiellement réduite par le mode d'emploi que constitue la foi et par la famille en prière que forment les frères et sœurs en croyance.

8 Chaque partie de l'ouvrage illustre ce cheminement. L'aspiration individuelle à la modernité, à l'autonomie personnelle, à l'individuation se heurte à l'environnement. L'invitation permanente qui nous est faite de demeurer attentif au contexte dans lequel ce pentecôtisme s'inscrit recoupe la volonté d'identifier un groupe de la sortie du groupe, une manière pour l'individu naissant de s'accrocher à une collectivité lui permettant de battre en brèche les contraintes de la collectivité qui lui semblent l'étouffer. L'exemple du mariage est à cet égard très éclairant. La description qui nous en est donnée montre que les transformations à laquelle les "Américains » le soumettent sont importantes; le libre choix, le consentement mutuel en deviennent les bases, le mariage deviendrait, à les suivre, un simple contrat entre personnes. Dans le même temps, par delà les évidences énoncées par les protagonistes, la négociation sociale de l'acte n'a pas tellement changé dans son processus. Si la forme - au premier rang de laquelle l'opposition affirmée aux 
logiques traditionnelles et coutumières - subit une métamorphose, le principe même de l'échange entre groupes demeure. Pour prendre un second exemple, les croyantsguérisseurs qui sont évoqués dans un passionnant chapitre, mobilisent dans une vulgate protestante les formes traditionnelles de la sorcellerie : " A travers une subtile interpénétration de ces deux dispositifs de guérison apparaît une double scène. Elle mêle la doctrine officielle des Assemblées de Dieu [...] avec les subtils arrangements quant au rapport à la loi, ouverts par la conception d'un 'moi multiple' révélé ici par le génie de la possession » (Laurent, 2003 : 348). L'existence d'un moi multiple, qui subsiste dans la vision protestante, même si ses instances y sont renommées de noms plus bibliques, invite à déresponsabiliser les personnes et à chercher les causes du mal-être dans l'envie ou l'attaque d'autrui. L'interprétation classique demeure, et ainsi demeure compréhensible pour tous, alors qu'en même temps "l'invention de solutions individuelles, situées à la croisée des principes de la sécurité de la 'concorde coutumière' et des principes de la 'concorde civile' » (ibid. 349) $)^{5}$ deviennent possibles.

Comme Laurent l'évoque pour le mariage, "les anciennes formes d'alliance ne disparaissent pas mais plutôt survivent [...] en filigrane des formes d'alliances par consentement mutuel religieux " (2003 : 97), la tradition s'invite au centre même de la modernité. A quelques endroits, l'auteur parle à ce propos de bricolage. S'il est bien question de faire du neuf, ou du "fonctionnant nouvellement", en recomposant les éléments de différentes traditions auxquelles la société burkinabée est soumise, la sienne évidemment mais aussi toutes les influences exogènes, l'image conserve du sens. Mais il me parait que les recompositions vont bien plus loin. Il n'est pas question de faire du neuf avec du vieux, ou de l'inusité ; au contraire, il est question de se ressourcer, de bâtir de l'inédit sans renier mais en métamorphosant l'existant. Comme l'ensemble de l'ouvrage le montre, il n'est pas de remise en cause mais une reconstruction où tous les éléments qui ont servi à la fabrication, à la naissance, se reconnaissent mais changent de statut. Ils sont toujours présents mais n'appartiennent plus aux différentes traditions qui les ont originairement produits. Dans ce sens, l'hybridation (Gruzinski, 1999) est un concept central de ce travail. Les influences se sont unies pour produire du neuf, c'est-à-dire quelque chose qui n'est pas tout à fait le produit visé et plus tout à fait celui de départ.

Plutôt que du pentecôtisme au Burkina Faso, Pierre Joseph Laurent nous entretient dans cette perspective des stratégies religieuses d'individus confrontés à la transformation de leur environnement local et à la difficulté de rencontrer leurs aspirations personnelles. Ces stratégies religieuses conservent - voire accroissent - une vigueur importante dans le monde actuel: l'Afrique est à cette enseigne exemplaire (faut-il évoquer le cas du foisonnement des Eglises dans la République démocratique du Congo?). La foi offre le socle raisonnable pour affronter le monde qui est vraiment insécurisé. Malgré la sécularisation endémique de notre Europe, de telles stratégies s'y observent également, peut-être de manière plus marginale. Si pour ces fidèles - qu'ils soient les fidèles des "Américains » au Burkina ou ceux du Père Samuel à Charleroi - la religion et la foi sincère sont des prétextes, des outils leur offrant la possibilité d'affronter le monde moderne, ontologiquement incertain. Il nous faut lire ce travail dans cette perspective, en cherchant à saisir comment ils utilisent leurs croyances, tout en étant évidemment manipulés par elles, pour sortir de leur condition, pour réintroduire de la certitude dans leur monde. Ne nous trompons pas dans la lecture : malgré le titre de ce livre, l'ouvrage n'est pas le recueil de l'histoire d'un mouvement, il traite de l'entrée en modernité de ces 
croyants, de l'action de ces gens, des hybridations qu'ils réalisent pour continuer à (sur)vivre dans leur contexte local.

\section{BIBLIOGRAPHIE}

$\mathrm{BECK}$, Ulrich, 2001, La société du risque. Sur la voie d'une autre modernité, Paris : Aubier, Alto, traduit de l'allemand par Laure Bernardi (Risikogesellschaft, 1986), préface de Bruno Latour.

BOLTANSKI, Luc et THÉVENOT, Laurent, 1991, De la justification. Les économies de la grandeur, Paris : Editions Gallimard, NRF.

BOURDIN, Alain, 2000, La question locale, Paris : Presses Universitaires de France.

CHARLIER, Jean-Émile et PIERRARD, Jean-François, 2001, « Systèmes d'enseignement décentralisés dans l'éducation sénégalaise, burkinabée et malienne», Autrepart, 17, Des écoles pour le Sud, IRD, Paris.

DURKHEIM, Emile, 1897, Le suicide, Paris : Presses Universitaires de France, $7^{\mathrm{e}}$ édition de 1993.

GRUZINSKI, Serge, 1999, La pensée métisse, Paris : Librairie Arthème Fayard.

LAURENT, Pierre-Joseph

1998, Une association de développement en pays mossi. Le don comme ruse, Paris : Editions Karthala.

2003, Les pentecôtistes du Burkina Faso. Mariage, pouvoir et guérison, Paris : IRD Editions et Karthala.

MOENS, Frédéric, 2002, « Expérience religieuse. D'anciens cadres pour un nouvel imaginaire », Sociétés. Revue de sciences humaines et sociales (Demeures de l'humain), 75/1, pp. 41-51.

RIST, Gilbert, 1996, Le développement. Histoire d'une croyance occidentale, Paris : Presses de la Fondation Nationale des Sciences Politiques.

\section{NOTES}

1. Références par ailleurs diabolisées, il n'est pas une région d'Afrique où l'origine paysanne ne soit pas remise en cause, occultée, rejetée comme le temps d'un travail dur, ingrat et sans avenir. L'ambition est au fonctionnariat et au travail citadin ; les portraits physiques que dresse PierreJoseph Laurent - où les individus se présentent toujours en complet-veston, chemise à manches longues, stylo-bic dont l'onglet est visible au revers d'une poche - en sont la parfaite expression, celle de la rupture paysanne. Le cas exposé au Burkina est à cet égard impressionnant. Mais il n'est en rien particulier: de nombreuses recherches montrent la place que ce rejet d'une condition paysanne prend dans les comportements africains actuels.

2. Loin d'être un spécialiste du pentecôtisme des Assemblées de Dieu, je n'ai eu l'occasion que de les rencontrer dans le cadre de quelques missions au Burkina, il est frappant de constater, dans l'usage fait de ce terme, une convergence étonnante avec l'Opus Dei dans nos régions du Nord de la Méditerranée. 
3. Cette observation est centrale dans l'analyse actuelle des pays africains. Elle se retrouve dans l'analyse portée sur les systèmes d'enseignement (Charlier et Pierrard, 2001). La démission d'Etats soumis aux contraintes de l'ajustement structurel et l'impossibilité pour les ordres traditionnels de gestion du collectif de poursuivre leur travail est probablement un des éléments centraux de la crise actuelle, une situation que Laurent décrit - à la suite de de Certeau et de Sibony - comme étant le résultat d'un " entre-deux ", une impossibilité très inconfortable de se situer et d'agir dans un cadre de référence cohérent et anticipable par tous.

4. Il y a presque vingt ans, Beck soulignait déjà pour l'Occident les difficultés, les dangers et les enjeux de ces sociétés modernes intégrant tous les types de risque, acceptant en d'autres termes que le calculable fasse irruption dans le quotidien (Beck, 2001). Ce refus radical de l'incertitude inhérente à la vie et l'acceptation irraisonnée du risque (qui est une version affadie de l'incertain car y est préférée l'anticipation statistique d'une catastrophe à l'inconnu de sa réalisation) se retrouvent sous une autre forme dans la société décrite: le rejet des solidarités mécaniques jugées par trop contraignantes et l'impossibilité de leur substituer des solidarités organiques efficaces impose d'accepter un risque que les stratégies protestantes, mais d'autres également, permettent d'assurer quelque peu.

5. La concorde civile est, pour Laurent, le pendant moderne de la concorde coutumière. Il souligne que la situation moderne, caractérisée entre autres par l'accumulation pour soi et non plus pour le groupe, en d'autres termes la satisfaction des intérêts individuels, suppose l'édification d'un espace public garantissant pour tous une sécurité minimale. Dans cette version « libérale », la confiance doit y être garantie par une instance supérieure, matériellement l'Etat : « Dans la 'concorde civile' l'assouvissement de l'intérêt individuel implique l'établissement d'un accord qui conduit les personnes à s'élever au-dessus de la contingence pour la gestion de biens communs dans l'intérêt de tous " (Laurent, 2003: 270). Cette vision, optimiste, évoque les axiomes que Boltanski et Thévenot (1991) définissent pour qu'une cité puisse être définie. Mais dans l'âpreté et la violence de la société burkinabée, comment la confiance pourrait-elle dépasser la sphère contrainte de la famille?

\section{AUTEUR}

\section{FRÉDÉRIC MOENS}

Frédéric Moens est sociologue et anthropologue, chargé de recherches aux Facultés universitaires catholiques de Mons (FUCaM). Il enseigne au Conservatoire royal de Bruxelles, à l'Institut des Hautes Etudes des Communications Sociales et aux FUCaM. Sociologue des religions, ses travaux concernent la place que le catholicisme occupe dans les sociétés européennes.

Actuellement, il mène des recherches en sociologie de l'éducation. 